\title{
BILDU: Compile, Unify, Wrap, and Share Digital Learning Resources
}

\section{Oskar Casquero, Javier Portillo, Manuel Benito, and Jesús Romo Universidad del País Vasco / Euskal Herriko Unibertsitatea EUITI Bilbao, Bilbao, Spain}

\author{
oskar.casquero@ehu.es javier.portillo@ehu.es \\ manuel.benito@ehu.es jesus.romo@ehu.es
}

\begin{abstract}
The Internet has become an unstructured database where it is difficult to obtain relevant and quality results. This fact hinders the collection of digital resources that can be used for educational purposes. Besides, learning resource harvesting tools neither refine search results based on user profile information, nor do they facilitate collaboration between users with similar interests. Overcoming these limitations is very important in order to find, retrieve, and share learning resources. This paper gives an overview of current search technologies and proposes to develop a social search framework that would adapt generic search paradigms to the specific characteristics of learning resources. The idea of the framework is based on the following concepts: compile (indexation of learning resource repositories and creation of vertical search engines), unify (integration of vertical search engines), wrap (designing the appropriate interfaces that fetch search criteria and present results), and share (building social networks around users and search results).
\end{abstract}

Keywords: learning resource, information source, vertical search engine, preference queries, multi-form results, collaborative filtering, loosely coupled services.

\section{Introduction}

The reuse of existing learning materials is an important topic in current research (Zimmermann, Meyer, Rensing, \& Steinmetz, 2007) where learning objects play an essential role. One of the problems that must be overcome for the normalization of learning objects utilization is the difficulty of finding them (Bates, 2005). Obviously, search engines can solve the problem of seeking and retrieving learning objects, but there is a lack of suitable search engines in this field.

Nowadays, the use of search engines has widely contributed to the success of new learning processes, e.g. thanks to search engines users can carry out their own personal research to collect learning materials. But, despite the Internet providing an inexhaustible amount of learning mate-

Material published as part of this publication, either on-line or in print, is copyrighted by the Informing Science Institute. Permission to make digital or paper copy of part or all of these works for personal or classroom use is granted without fee provided that the copies are not made or distributed for profit or commercial advantage AND that copies 1) bear this notice in full and 2) give the full citation on the first page. It is permissible to abstract these works so long as credit is given. To copy in all other cases or to republish or to post on a server or to redistribute to lists requires specific permission and payment of a fee. Contact Publisher@InformingScience.org to request redistribution permission. rials, the shortage of learning objects within all the learning resources available on the Internet hampers the effectiveness of learning object search (Taibi, Gentile, \& Seta, 2005). Concepts of learning object and learning resource should be distinguished.

For the purposes of this paper, we define learning object as an independent digital resource packaged in accordance with the rules set by any of the international 
standards (e.g. SCORM, IMS, or LOM), while we define learning resource as any digital content that could be used for educational purposes (e.g. bibliographic references, research papers, book chapters, multimedia material, and learning objects).

Figure 1 represents the percentage of each learning material type in the repositories indexed by OpenDOAR (http://www.opendoar.org). The chart shows that only $13 \%$ of all the learning resources are learning objects ("Content Types," 2007). Therefore, it can be assumed that learning objects are a small part of learning resources.

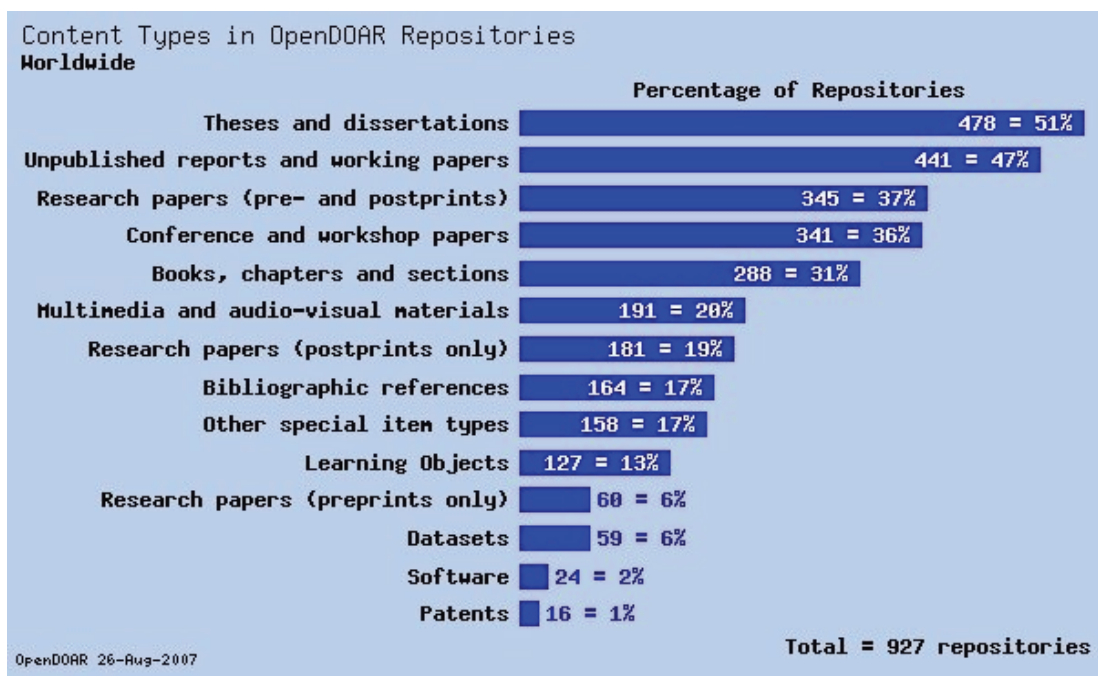

Figure 1: Relative importance of learning objects within the whole learning resource set.

As learning objects are based on learning resources, we think that the improvement in the search of learning resources is a prerequisite to facilitate the creation and reuse of learning objects (Portillo, Romo, Benito, \& Casquero, 2007). However, when trying to find learning resources, current search engines have the following limitations:

- Great amount of non-meaningful results. It requires some time to filter which digital resources really relate to learning resources; i.e. the number of results is not indicative of quality, e.g. a broad Google search delivers a very large number of resources of low value, whereas an Ovid search (http://www.ovid.com), while only returning one result, can point to a resource of very high quality.

- Lack of specific indexes for learning resource repositories. There are a great number of information sources related to e-learning on the Internet, and it is necessary to assess the quality and reliability of the material they contain.

- Need for more advanced user interfaces to launch searches and present results. The traditional text box where keywords are typed and the usual list of hits returned by a search engine should be enhanced. With respect to queries, the use of a more advanced syntax does not always help to reduce the large number of returned results. Regarding the list of results, ranking mechanisms try to sort them and show best matches first, but this notion of relevancy is typically a score computed out of elements (e.g. number of occurrences of a keyword, proximity of keywords, etc.) that do not necessarily represent user preferences (Abel, Herder, Kärger, Olmedilla, \& Siberski, 2007). Besides, there is not usually information about relations among different results.

- Shortage of social networking services. Search engines do not facilitate collaboration between people with similar interests. 
This paper focuses on the whole set of learning resources, rather than on the subset of learning objects, and explores solutions to enhance access to digital resources that are likely to be used as learning resources by various communities.

The rest of the paper is laid out as follows: first, a set of actions is proposed that should lead to achieve the aforementioned goal; next, we describe an architecture for a search framework prototype that will be used to validate the ideas derived from those actions; finally, conclusions and future work are presented.

\section{Action Lines}

All the information about this ongoing project can be accessed in the blog REAPRENDIENDO (http://reaprendiendo.blogspot.com). This blog is the space where we collect ideas, reflections, news, trends, and tools that could help to adapt the paradigms used in search engines, such us resource selection and presentation, to the needs of learning resources.

Four action lines guide the research on the project and organize the information in the blog:

- Compile. It explores the creation of vertical search engines. It is based on indexing repositories, archives, wikis, blogs, and other websites linked to learning resources. Information about this action line is collected in the blog under the tag repo.

- Unify. It covers the integration of vertical search engines in terms of new retrieval techniques, new search engines, and new ways to relate those search engines. Information about this action line is collected in the blog under the tag search.

- Wrap. It analyzes the design of appropriate interfaces to interact with users when a search is launched, e.g. displaying the results of a multiple search in an interrelated way. Information about this action line is collected in the blog under the tag interface.

- Share. It considers the construction of social networks around users and search results. It is based on new collaborative mechanisms added to search engines. Information about this action line is collected in the blog under the tag social.

This set of ideas will assist the design of BILDU (a word in Basque which means compile, unify, wrap, and gather), a search framework prototype that is intended to validate these concepts. With

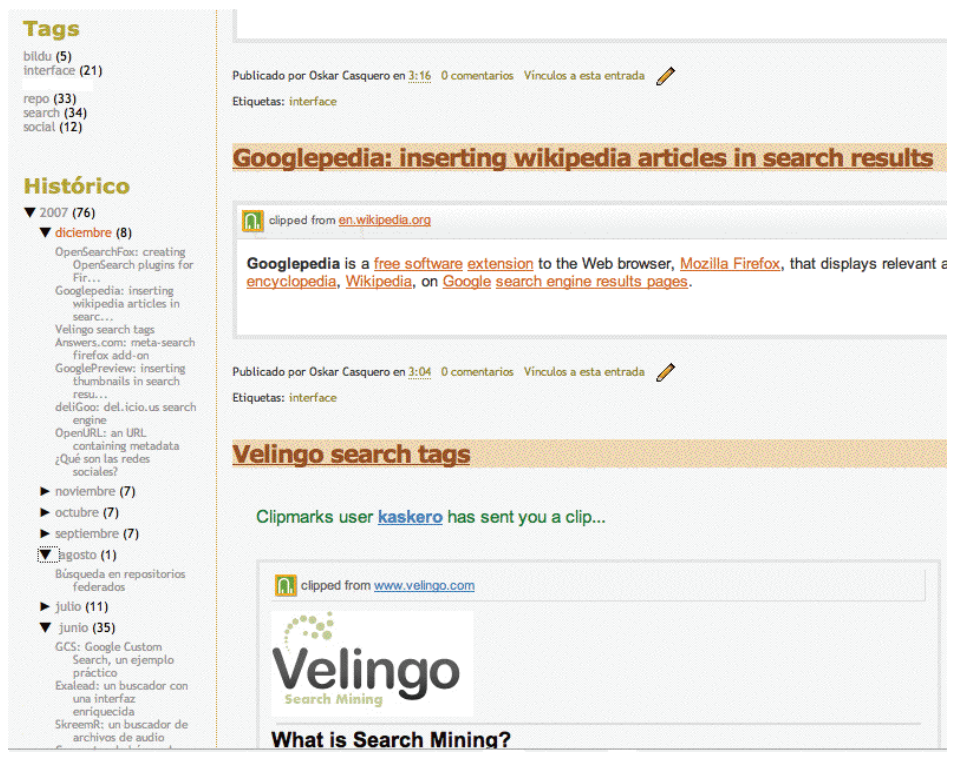

Figure 2: A screenshot of REAPRENDIENDO blog. 
the aim of documenting changes as they occur in BILDU, there is one final tag bildu. Figure 2 shows a screenshot of the blog.

The following subsections of the paper summarize how the information we have started to write on the blog can help to identify repositories that will be indexed (compile) in BILDU, where several search engines will be integrated (unify) with enhancements in their interfaces (wrap) and social networking features (share).

\section{Compile (repo): Indexing Information Sources}

Much of the work in e-learning is focused on the supplier side, i.e. how to generate materials and make them available. In this sense, there are many repositories and archives distributed across the Internet which store thousands of free access digital resources of an academic nature. Moreover, the so-called Web 2.0 has involved the consolidation of three key services as carriers for the shared knowledge: wikis, blogs, and social networks.

However, simply making learning materials available does not help to deliver learning materials to the consumer side (Roberts, 2005). Learning materials are heterogeneous (differences in topics, formats, and licences) and information sources are independent of each other (differences in technical standards and supporting platforms).

Reflections derived from interoperability standards state that "Finding content, when there are multiple repositories of content to be searched, is a complex problem. The problem is further aggravated when the repositories have heterogeneous representations of metadata and heterogeneous access methods" ("IMS Digital," 2003).

On one hand, due to their distribution, it is difficult to know the existence and location (web address) of information sources. Access to resources can be a tedious task for users if they have to seek, find, and evaluate useful information sources. On the other hand, due to their heterogeneity, it is difficult for users to cope with the particular characteristics of each information source, for search engines to accomplish queries from each information source, and for information sources themselves to share and exchange data between them.

In summary, despite there being a large and growing body of learning materials available from several information sources over the Internet, there is not a central and homogeneous access to those information sources. Our proposal aims to unlock these materials for the learning community.

A main challenge in delivering high quality learning resources is the provision of an infrastructure that is capable of compiling information sources and their features. Thus, careful consideration must be given to access modes, resource discovery, and end users.

In order to achieve this goal, BILDU covers the task of searching, cataloguing, and indexing all the information sources that are susceptible of being interesting for different learning contexts. This task is being carried out through the REAPRENDIENDO blog. The information contained in the blog will allow drawing a map of sources properly classified according to:

- the type of resources stored or referenced by the sources, e.g. public domain texts, free photographs, educational images, interactive learning objects, etc.

- the access protocols for interacting with the sources, e.g. Z39.50, OAI-PMH, open APIs, REST, SOAP \& XQuery, SOAP \& XPath, etc.

- the metadata schemas retrieved from the sources, e.g. IEEE Learning Object Metadata (LOM), IMS Resource List Interoperability (RLI), Dublin Core (DC), etc. 


\section{Unify (search): Integrating Vertical Search Engines}

In general, a user who looks for a resource makes a query to a search engine. The user needs several queries so he can refine obtained results until they are satisfactory. This process may require some patience and does not ensure finding an appropriate resource that meets user requirements. In order to find properly filtered quality resources, it is necessary to use alternative search procedures that will ensure a high retrieval and precision capacity.

There are different techniques that help to define the content and the context of a search and make it easier for users to find information that matches their search criteria:

- Specific search engines. Some search engines look up a limited number of sites on a particular topic, allowing a more efficient information retrieval. As they deal with a smaller number of sources than a generic search engine, these specific search engines can update more frequently the sources of the indexed sites.

- Personalized search engines. Their main feature is the creation of custom search engines by means of the restriction of the search scope to a list of websites to which different ranks can be assigned. This type of search engine also allows social networking thanks to collaborative mechanisms that allow their users to assess the websites in the list and enrich the search scope by suggesting or adding new sites.

- Social search engines. They store references to resources collected and labelled by users. This human filter reports a great precision as labels let users define the metadata that describes content and interests of a community. Furthermore, the use of tags improves the effectiveness of the search and promotes the creation of spontaneous social networks.

- Semantic search engines. The problem with current search engines is that they can only find resources that match the search criteria in content or metadata, e.g. if a search on Electronic Devices is launched, search engines will only retrieve results which have the words Electronic and Devices on them; resources regarding Semiconductors, Diodes, Transistors or Integrated Circuits will not be retrieved as search results. Semantic search will solve this problem by analyzing not only words, but their meaning and the correlation between them as well, i.e. the way they are semantically connected.

It must not be forgotten that no matter how good any of the above options could be, they are never going to cover a very high percentage of the whole Internet. Hence, sometimes it would be necessary to use several of them at the same time to ensure sufficiently precise and comprehensive results. There are different approaches to achieve this objective:

- Federated search across search engines. Search engines implementing this type of meta-search contain records of other search engines. When a user launches a search, it is forwarded to linked search engines. Then, all the results are returned together as a whole.

- Federated search across databases. This type of meta-search builds a united platform where heterogeneous databases can be concurrently inspected and provides a consistent result display by integrating search results from each database.

There are several initiatives that promote the development of new information retrieval systems that allow an easier access to academic and scholar learning resources. Let us mention the most significant of them.

One of the first initiatives for learning resource search was led by Elsevier. This publisher devised a search engine capable of indexing web pages automatically, as generic search engines do, but filtering information so that it could be acceptable for the strict scientific criteria. The product was called Scirus (http://www.scirus.com). 
Scirus returns results from the whole Internet, including access-controlled sites that other search engines do not index. In addition to web pages, Scirus indexes special scientific information sources, provided by both commercial and open source publishers, which otherwise would not be accessible.

Following a similar procedure, Google and Microsoft launched their own academic and scientific search engines, known as Google Scholar (http://scholar.google.com) and Live Search Academic (http://academic.live.com), respectively.

The latest ScientificCommons (http://www.scientificcommons.org), like the veteran OAIster (http://www.oaister.org), makes use of Open Archives Initiative Protocol for Metadata Harvesting (OAI-PMH) to index many institutional repositories and open archives. OAI-PMH is a lowbarrier mechanism for the interoperability of information sources that allows search engines to harvest metadata exposed by repositories ("Open Archives," 2004).

More recently, there have been other proposals based on personalized search engines, among which OpenDOAR deserves special attention. OpenDOAR stands out for having one of the greatest indexes of existing repositories. Thanks to Custom Search Engine (CSE) technology of Google Co-op, OpenDOAR can launch simultaneous searches on those repositories. Furthermore, OpenDOAR offers an API that allows its integration in federated search environments.

Nowadays, the rise of social search engines is foreseen (Portillo, Benito, Romo, \& Casquero, 2006). Wikia Search (http://search.wikia.com) is an example of this. The objective of Wikia Search project is to build a social network that values web resources, as it is based on the idea that human beings are better than generic search engines to give an opinion about an information source.

deliGoo (http://www.deligoo.com) is another example of search engine that shares the social search idea. deliGoo creates a powerful Google CSE on demand which indexes the del.icio.us bookmarks of the user. Moreover, it also offers the ability to search using not only bookmarks, but also a certain tag used by many del.icio.us users, thus expanding the search scope to the websites bookmarked with that tag.

Current approaches towards enhancing reusability of learning materials agree that search engines will eventually include semantic support. Nevertheless, it is surprising that none of the aforementioned initiatives have considered including some of the aspects of the Semantic Web, such as the use of ontologies (Codina, 2007). Although the implementation of semantic features is a hard task, there have been several tries in different universities that show that it is possible to develop ontology-based search engines for learning objects (Soto, García, \& Sánchez-Alonso, 2007). Perhaps it is too soon to start thinking of unifying, and different types of search engines would probably have to mature separately before they decide to join forces.

As it can be seen, the evolution of search engines comprises several trends:

- The selection, cataloguing, and indexation of information sources.

- The concurrent search among different information sources and search engines.

- The personal content filtering based on user preferences.

- The collaborative content filtering based on community interests.

- The spreading of the search over semantically related resources.

Considering current search technology developments and future trends, a search framework must be built keeping in mind openness and value-added user experience. Therefore, the BILDU 
search framework prototype should be designed as a unique interface that hides the complexity of the search process and simplifies the interaction with the search environment of the user.

This unique interface is responsible for integrating the so-called vertical search engines, which are different search services based on the types of search engines described before. Vertical search engines will specialize in particular tasks: different types of licenses, different types of files, and so on. The set of vertical search engines will consist of two service subsets:

- Own services: small personalized search engines based on the enriched indexing that results from the compilation of information sources relevant to life-long learning.

- Outsourcing: specific and social search engines that can be accessed through standards or lightweight proprietary APIs, and their integration in the framework as web services.

Although vertical search engines will be useful by themselves, BILDU should offer federated search capabilities that allow simultaneous resource retrieval from several information sources.

BILDU should be a social environment that connects the user to individual and cooperative search decisions throughout the Internet. This feature can be achieved by building a preferencebased system that exposes user interests to the community in a variety of ways and allows automated but selective search of digital learning resources.

Finally, it would be desirable to take into account the integration with other ontology-based search engines. Thus, when launching a search, BILDU would be capable of offering collections of resources with the same semantic profile as the one specified in search keywords.

\section{Wrap (interface): Search Interface and Results}

Search engine users can spend a great deal of time attempting to find what they are looking for because they are either not using the correct search term or the most relevant result is hiding after the first results page; they keep searching, try a different search engine, or give it up.

The objective of BILDU is to provide a simple interface that gives fast access to the available learning resources, guiding the user to the resource that best satisfies her and merges selected resources in a single list of results based on user preferences.

Common search engines lack a suitable interface in order to specify search criteria. This is due to the fact that a search is not well supported by a query model where users can only specify hard constraints, e.g.: too specific query predicates often lead to empty result sets, while too unspecific query predicates may yield a huge number of results (Orzechowski, Ernst, \& Dziech, 2007). Moreover, search engines do not usually remember our usual selection criteria for searches.

In order to overcome these problems, capabilities of search interfaces have to be improved through the introduction of semantic-based queries and preference-based queries:

- Semantic-based queries are an efficient way to enter selection criteria. The user enters a complete question that is understood by the search engine, which spreads the search scope over semantically related terms and gives an answer in terms of an accurate list of results.

- Preference-based queries can be used in order to increase the expressivity of queries. This helps users to describe more accurately their interests and retrieve efficiently optimal matches according to their preferences.

Experimenting with semantic and preference based search in BILDU will allow users to influence their own search experience by adding, moving and deleting search results. Besides, BILDU will 
also allow establishing permanent and automatic custom search channels through the use of syndication services where user preferences would be loaded from their profiles.

The layout of the results based on a flat list of links is not the best suited to facilitate the selection of learning resources. Most of the current search engines rarely introduce abilities to connect the results or suggest new searches based on previous concepts. A good search engine must present search results in different forms in order to activate as many senses as possible (Linckels \& Meinel, 2004) because information that is presented at the same time in different forms improves its understanding (Roberts, 2005).

This problem could be solved by organizing the results along with others conceptually related through conceptual maps, as WikiMindMap (http://www.wikimindmap.org) does, or clusters, as Vivísimo (http://vivisimo.com) does. Figure 3 shows screenshots of these examples.
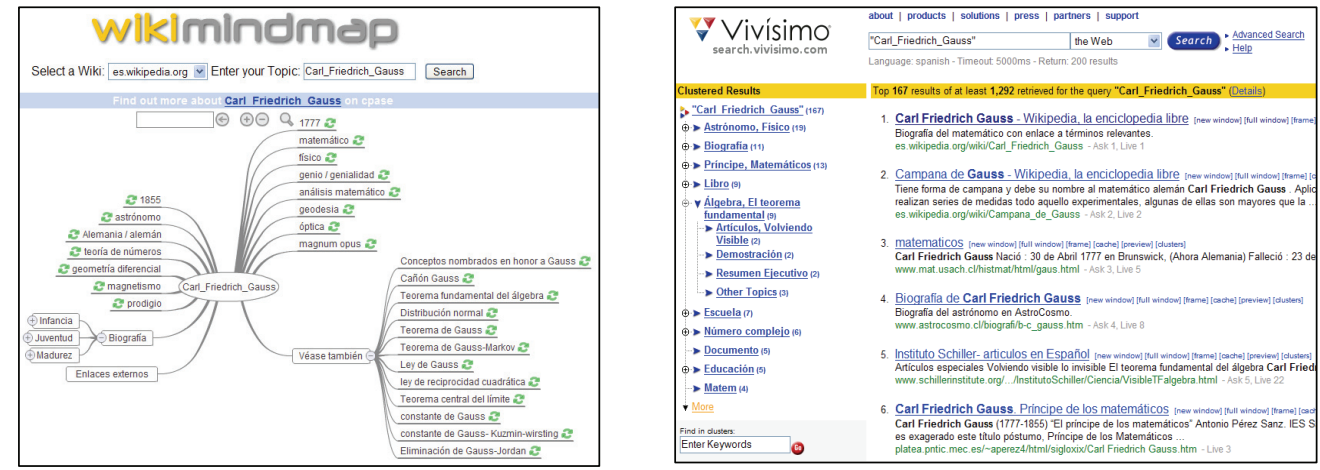

Figure 3: WikiMindMap (conceptual maps) and Vivísimo (clusters) screenshots

In addition, resource previews can also be very useful for the selection of the best search results. There are several software solutions that offer resource preview capabilities. Two of them, in the form of Firefox add-ons, are mentioned here: Cooliris Previews, which lets the user preview links and rich media without leaving search results page, and Google Previews, which inserts preview images of websites into the Google and Yahoo search results. Figure 4 shows a search result page that includes a preview of the first result together with thumbnails for each of the results.

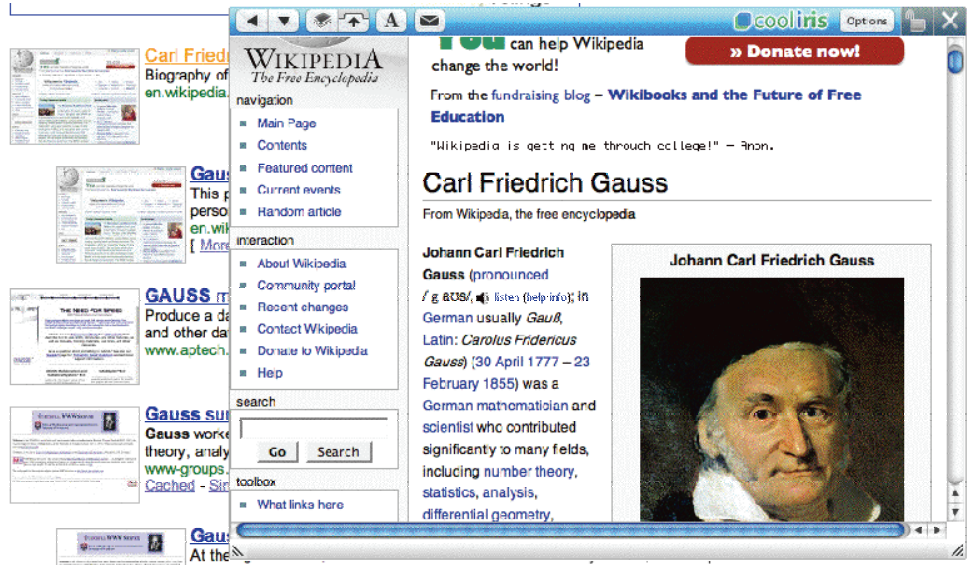

Figure 4: A search result preview together with thumbnails (on the left) for each result

As search engines are moving from a monolithic design to a more open and distributed architecture, the aforementioned features that enrich user experience should be part of the core capabilities of a search engine. A suitable approach for including multi-form results and resource previews in BILDU should consider their implementation as rich client side tools that are easily plugged into the search framework. 


\section{Gather (social): Building Social Networks}

People who retrieve and reuse learning resources form a heterogeneous group, but they share some concerns and needs. Hence, it is interesting for search engines to provide a flow of information among these people, to help them to discover each other - ultimately, to create social networks spontaneously.

Most of the search methods used in Internet match query keywords with the metadata of the available resources and produce a list of results. When query conformity is not enough to sort search results properly, search engines use various generic schemes to arrange those results. As people evaluate things on a very subjective basis, it is impossible to provide a homogeneous ranking scale that is applicable for all users (Orzechowski et al., 2007).

However, despite the preferences of each user being different, groups of people have common interests. If users could make an assessment of a given search result according to their preferences, search engines could sort result lists based on the ranks assigned by those users who have, with a large degree of probability, similar interests.

Since search engines are not accurate enough, human feedback must be considered as a part of search engine decisions. At this point, collaborative filtering becomes essential. Collaborative filtering allows search engines to predict user affinity for information by connecting user recorded interests with the recorded interests of a community and sharing ratings between likeminded users (Herlocker, Konstan, \& Riedl, 2000).

There are two commonly known mechanisms for collaborative filtering:

- Ranking: when the user applies a rank within a given scale to a resource or website.

- Tagging: when the user attaches a label to a resource.

Ranking and tagging are used to assess user preferences with regard to a set of items. Both of them are active methods because they involve an explicit action from the user who assesses a resource. These methods have the advantage of improving the reliability of collected opinions. Their main disadvantage is that active methods require user input.

Ranking is based on the fact that the evaluation of search results by a user can help result selection for other users. This means adding voting features that allow users to value the search results they see. Ranks also promote those resources that best satisfied user wishes. As several web services like Digg (http://www.digg.com) have proved, ranking can successfully change the way people consume information.

Digg is a well known example of a web service where people use ranking to collectively determine the value of a resource. If a resource receives enough votes, it is promoted in the front page for visitors to see it. Within its Experimental Labs (http://www.google.com/experimental), Google is playing with Digg style ranking ("Google Experimental," 2007). Figure 5 shows a screenshot of a Google search result page to which Digg style buttons have been added. 


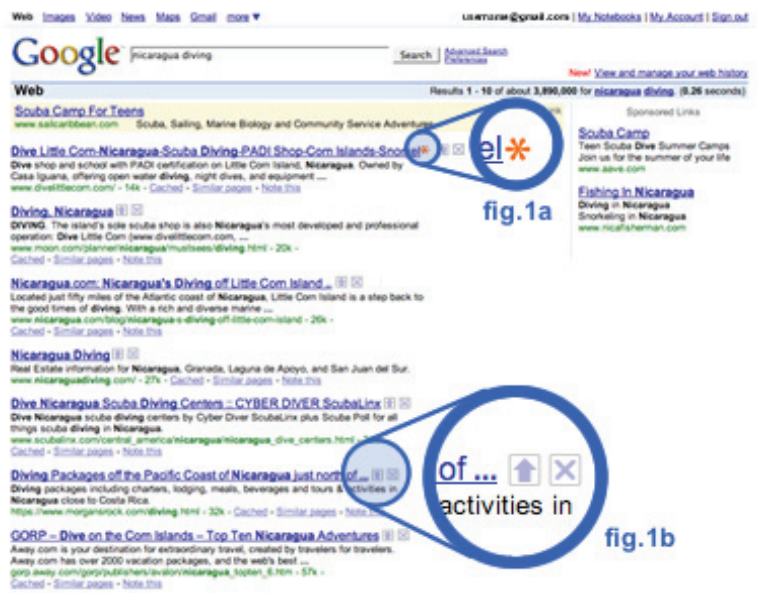

Figure 5: Google's Digg style experiment

Tagging is based on the fact that any user is part of the overall search experience. Tags can help users in the following tasks:

- Finding similar results labeled with the same tags.

- Expanding or narrowing searches, filtering best results.

- Learning from others, suggesting better keywords.

An example of search tagging software can be found at Velingo SearchTags, a Firefox extension that enhances Google, Yahoo, and Live search results by adding tags to search result pages. Tags are built automatically from a global social knowledge which is constantly updated by analyzing search logs and search session events. Decisions taken during the search sessions of each user add knowledge to the community and help other users. Figure 6 shows a screenshot of a search result page populated with Velingo tags.

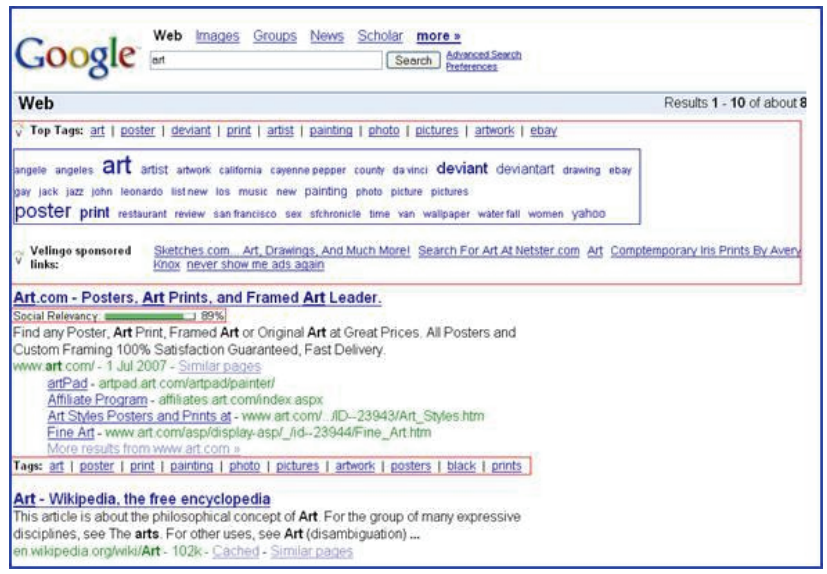

Figure 6: A Google search result page populated with Velingo tags

The design of BILDU must take into account the aforementioned mechanisms of ranking and tagging in order to collectively determine the value of a learning resource and create new sources of information to which users could subscribe through a syndication service.

Likewise, users should be able to suggest new sources for indexing in BILDU. Google already provides this feature in its CSE technology, and is also experimenting to open it to general search. Figure 7 shows the "know of a better webpage" feature. 


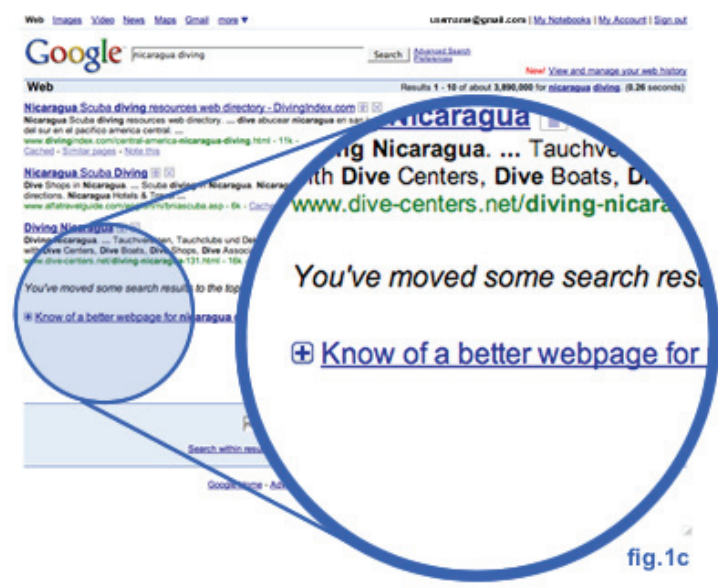

Figure 7: Users must be able to suggest the web sources that best suit search criteria

\section{Prototype Architecture}

Content is increasingly abundant and free. Currently Technorati claims to track 112 million blogs and over 250 million pieces of tagged social media ("Technorati: About," 2007). This is the sign of an emerging information overload that threatens to reduce the search interactivity of users. Furthermore, there are a great number of search engines across the Internet specializing in resources of different type and topic. A search framework like BILDU should allow focus, something like what a feed reader does.

Whereas a generic search engine operates within a global scope, BILDU operates at a personal level that coordinates information and services related directly to the user. However, BILDU can also consider the global scope as the range of services it can potentially integrate is not bounded within any particular organization or individual.

Because of this, the number of standards and protocols used to interact with third party services increases. It is no longer possible to focus solely on suiting the needs of a concrete provider. Instead, BILDU will need to interact with other services offering their own proprietary APIs (e.g. OpenSocial) and more general web standards (e.g. IETF Atom).

In addition, personal computing is currently in a state of transition. While traditionally users have mostly interacted with desktop applications, more and more of them are using web applications. Web applications are server side monolithic applications which are usually launched inside of a web browser. This means that they are unable to fully integrate with the computing environment of the user in order to take advantage of the benefits afforded to desktop applications.

Search engines are one of those web applications. But because of the continuous need to innovate, search engines must have an architecture that supports extension. Current developments show that search engines are going to evolve to a limited set of server side core capabilities into which rich search clients from many sources can be plugged (Severance, 2007).

Therefore, BILDU should not be designed as an application, but as a set of tools and services used in everyday life for searching different information sources. If different tools and services are going to be integrated, then open internet standards and lightweight proprietary APIs become critical. So, rather than a monolithic design driven search framework, BILDU is based on the idea of loosely connected small pieces. 


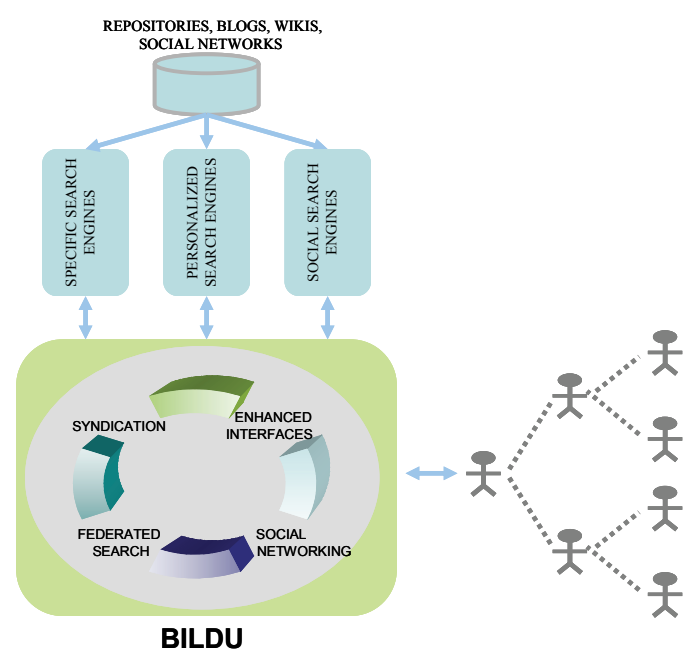

Figure 8: Architecture of BILDU.

As it can be seen in Figure 8, users interact with the framework through enhanced interfaces. These interfaces include capabilities to process preference-based queries, present multi-form results, and display resource previews. But user interaction is not only limited to search as the framework also offers tools that allow connecting the user with the interests of a community. Server side core features are built upon vertical search engines, which are composed of own and third party search services. Search services in turn, feed with academic and scholar information sources. Federated search makes possible the simultaneous search on various search services.

While third party search services are seamlessly integrated thanks to lightweight proprietary APIs, own search services are built upon Google Co-op technology (http://google.com/coop). Google Co-op is a platform that allows users to refine queries and feature specialized information in web searches. This is achieved by means of the creation of Custom Search Engines (CSEs) (http://google.com/coop/cse). CSE brings together categorization and indexation of websites with Google Web Search technology. Collaborative filtering is supported through Google Marker (http://google.com/coop/cse/marker) bookmarklet, which allows adding and labelling entire sites or URL patterns.

Unlike common search engines, BILDU is concerned with sharing resources for collaborative knowledge construction, and emphasizes the use of Creative Commons licences. In its initial stage, rather than pre-packaged learning objects, the resources collected and accessed using BILDU are more typically texts, images, sounds, videos, blog posts, comments, etc.

\section{Conclusions}

Learning resource discovery has been overlooked by current search engines. With the proliferation of repositories, archives, wikis, blogs, and social networks, the need for a unified, consistent and dynamic search of well catalogued and indexed information sources is a critical issue.

Moreover, with regard to user search experience, several strategies should be adopted: preference-based queries allow users to specify their wishes in order to obtain the best matches; multiform results introduce abilities to connect results and suggest new searches based on related concepts; resource previews enhance search result selection; and collaborative filtering allows prediction of user affinity for information and connects it with the interests of a community.

The architecture of the search prototype is a pragmatic approach that encourages a rapid development though the integration of vertical search engines. Instead of a large, rigid, and monolithic 
design, the selected solution is based on a small, loosely coupled and distributed service aggregation in order to reduce adaptation efforts in new search contexts.

Our future work focuses on the improvement of the current prototype and its integration with ontology-based search engines. The REAPRENDIENDO blog and BILDU prototype will allow us to perform simultaneous theoretical research and practical development work, respectively. This will give us the opportunity to test new ideas and acquire valuable experimental data.

\section{References}

Abel, F., Herder, E., Kärger, P., Olmedilla, D., \& Siberski, W. (2007). Exploiting preference queries for searching learning resources. 2nd European Conference on Technology Enhanced Learning. Lecture Notes in Computer Science, 4753, 143-157.

Bates, T. (2005). Questioning the value of re-usable learning objects in education: The need for a business case. Proceedings of II Simposio pluridisciplinar sobre diseño, evaluación y descripción de contenidos educativos reutilizables, Barcelona, Spain.

Codina, L. (2007). Search engines for scientific and academic information. Retrieved December 15, 2007, from http://www.hipertext.net/english/pag1021.htm

Content types in OpenDOAR repositories - worldwide. (2007). Retrieved December 15, 2007, from http://www.opendoar.org

Google Experimental Search. (2007). Retrieved December 15, 2007, from http://www.google.com/experimental/a840e102.html

Herlocker, J., Konstan, J., \& Riedl, J. (2000). Explaining collaborative filtering recommendations. ACM 2000 Conference on computer supported cooperative work. Retrieved December 15, 2007, from http://www.grouplens.org/papers/pdf/explain-CSCW.pdf

IMS Digital Repositories Interoperability - Core Functions Information Model. (2003). Retrieved December 15, 2007, from http://www.imsglobal.org/digitalrepositories/driv1p0/imsdri_infov1p0.html

Linckels, S., \& Meinel, C. (2004). A semantic search engine for a multimedia e-learning tool. Association for Educational Communications and Technology, UNT Summer 04 Conference. Retrieved December 15, 2007, from http://www.tele-task.de/en/paper/AECT-UNT2004.pdf

Open Archives Initiative Protocol for Metadata Harvesting. (2004). Retrieved December 15, 2007, from http://www.openarchives.org/pmh

Orzechowski, T., Ernst, S., \& Dziech, A. (2007). Profiled search methods for e-learning systems. First International Workshop on Learning Object Discovery \& Exchange. Retrieved December 15, 2007, from http://fire.eun.org/lode2007/lode08.pdf

Portillo, J., Benito, M., Romo, J., \& Casquero, O. (2006). GOXO: Un repositorio de objetos de aprendizaje basado en folksonomías. Proceedings of III Simposio pluridisciplinar sobre diseño, evaluación y descripción de contenidos educativos reutilizables, Oviedo, Spain.

Portillo, J., Romo, J., Benito, M., \& Casquero, O. (2007). Reflections on the next generation of authoring tools. Proceedings of the IADIS 2007 International Conference, Lisboa, Portugal, 2, 325-328.

Roberts, A. (2005). A future functional and technical specification for education media online. Report to JISC. Retrieved December 15, 2007 from http://www.jisccollections.ac.uk/uploaded_documents/EMOL Final Report_full.doc

Severance, C. (2007). The coming functionality mashup in personal learning environments. Retrieved December 15, 2007 from http://wwwpersonal.umich.edu/ csev/papers/2007/FunctionalityMashups_ver06.doc

Soto, J., García, E., \& Sánchez-Alonso, S. (2007). Semantic learning object repositories. International Journal of Continuing Engineering Education and Life-Long Learning. 17(6), 432 - 446. 
Taibi, D., Gentile, M., \& Seta, L. (2005). A semantic search engine for learning resources. Third International Conference on Multimedia and Information \& Communication Technologies in Education. Retrieved December 15, 2007, from http://www.formatex.org/micte2005/349.pdf

Technorati: About us. (2007). Retrieved December 15, 2007, from http://technorati.com/about

Zimmermann, B., Meyer, M., Rensing, C., \& Steinmetz, R. (2007). Improving retrieval of re-usable learning resources by estimating adaptation effort backhouse. First International Workshop on Learning Object Discovery \& Exchange. Retrieved December 15, 2007, from http://fire.eun.org/lode2007/lode07.pdf

\section{Biographies}

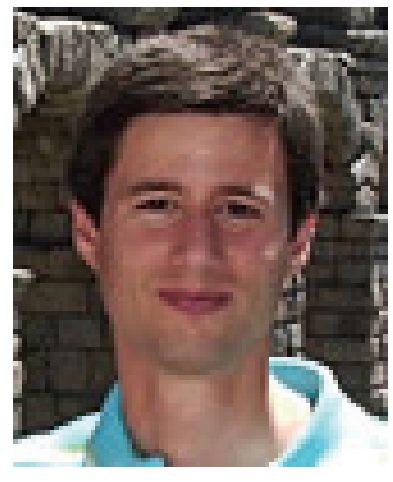

Oskar Casquero received his BS degree in Telecommunication Engineering from the University of the Basque Country in 2003. He works as Assistant Professor in the Department of Systems Engineering and Automatics at the University of the Basque Country. Before that, he has contributed, as Systems Analyst, to the design and development of a VLE (Virtual Learning Environment) for the same university. He is doing his $\mathrm{PhD}$ on Personal Learning Environments (PLEs).

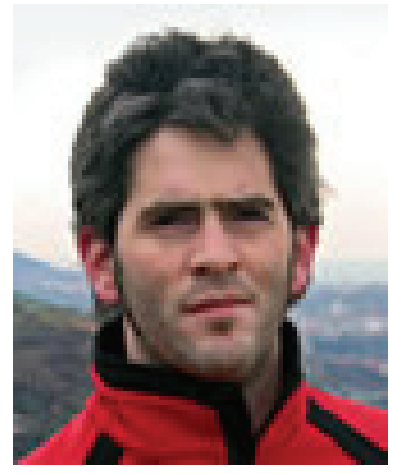

Javier Portillo received his BS degree in Telecommunication Engineering in 1997 and his PhD degree in Engineering in 2004, both from the University of the Basque Country. He works as Assistant Professor in the Department of Systems Engineering and Automatics at the University of the Basque Country. Despite he researched about Distributed Real-Time Control Systems for his $\mathrm{PhD}$, nowadays his research interests include PLEs and Authoring Tools for learning resources.

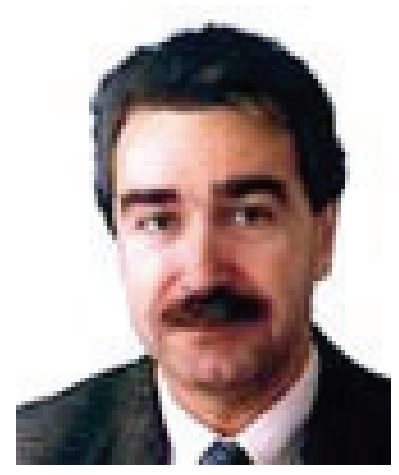

Manuel Benito received his BS degree in Mathematics in 1976 and in Psychology in 1984, and his PhD degree in 1993, all from the University of the Basque Country. He works an Associate Professor in the Department of Research Methods and Education Diagnosis at the University of the Basque Country. He is the assistant director of the Virtual Campus of the University of the Basque Country. His main research interests focus on training methodology for teachers in ICT. 


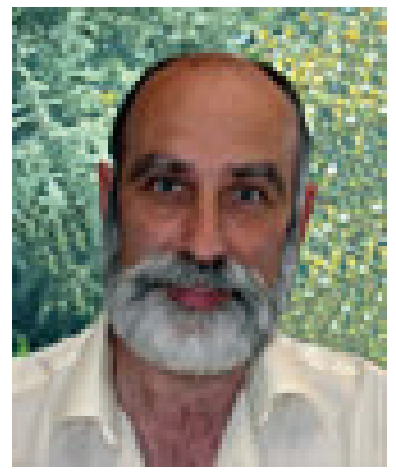

Jesus Romo received his BS degree in Industrial Engineering in 1986 and his PhD degree in Engineering in 1996. He works as an Associate Professor in the Department of Systems Engineering and Automatics at the University of the Basque Country. He is the director of the Virtual Campus of the University of the Basque Country. His research interests include VLE extension through functionality mashups, and improving reusability of learning objects. 